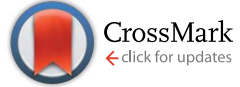

Cite this: Soft Matter, 2015, 11, 382

Received 3rd September 2014 Accepted 27th October 2014

DOI: $10.1039 / c 4 s m 01980 f$

www.rsc.org/softmatter

\section{Polymer conformations in polymer nanocomposites containing spherical nanoparticles $\uparrow$}

\author{
Argyrios Karatrantos, ${ }^{a}$ Nigel Clarke, ${ }^{\star a}$ Russell J. Composto ${ }^{\mathrm{b}}$ and Karen I. Winey ${ }^{\mathrm{b}}$ \\ We investigate the effect of various spherical nanoparticles on chain dimensions in polymer melts for high \\ nanoparticle loading which is larger than the percolation threshold, using molecular dynamics simulations. \\ We show that polymer chains are unperturbed by the presence of repulsive nanoparticles. In contrast \\ polymer chains can be perturbed by the presence of attractive nanoparticles when the polymer radius of \\ gyration is larger than the nanoparticle radius. At high nanoparticle loading, chains can be stretched and \\ flattened by the nanoparticles, even oligomers can expand under the presence of attractive \\ nanoparticles of very small size.
}

\section{Introduction}

The radius of gyration is fundamental to both structure and dynamics in polymeric systems. The addition of nanoparticles to a polymer matrix can result in materials with improved electrical, rheological and tribological properties ${ }^{1}$ relative to a polymer melt. In this paper, we explore how spherical nanoparticles affect polymer dimensions in nanocomposites in the cases when the polymer radius of gyration $\left(R_{\mathrm{g}}\right)$ is larger or of the order of the nanoparticle radius $(R)$.

There is controversy as to whether the addition of nanoparticles to a polymer melt alters the polymer conformation. In particular, neutron scattering of a polystyrene (PS) chains/ crosslinked PS nanoparticle $(R=2-4 \mathrm{~nm})$ nanocomposite ${ }^{2}$ showed a polymer chain expansion (20\% expansion of entangled polymers at nanoparticle volume fraction $\phi=10 \%$ ) for polymer chains with radius of gyration larger than the nanoparticle radius $\left(R_{\mathrm{g}} / R=1.6-5.7\right)$, which is contrary to recent studies of PS/silica $(R=6.5 \mathrm{~nm})$ nanocomposite ${ }^{3-5}$ for $R_{\mathrm{g}} / R=$ 1.9-3.9, ${ }^{5}$ and poly(ethylene-propylene) (PEP)/silica nanocomposite $(R=5-7.5 \mathrm{~nm})^{6}$ where the polymer chains were unperturbed. Moreover in a study of a poly(dimethylsiloxane)/ polysilicate $(R=1 \mathrm{~nm})$ nanocomposite, ${ }^{7}$ a significant increase of the polymer chain dimensions (reaching $60 \%$ expansion at nanoparticle volume fraction $(\phi=40 \%)$ ) was observed for $R_{\mathrm{g}} / R$ $=6-8$ (which is in agreement with the observations of Mackay ${ }^{2}$ ) and a decrease in polymer dimensions for $R_{\mathrm{g}} \approx R$. The quality of

${ }^{a}$ Department of Physics and Astronomy, University of Sheffield, Sheffield S3 7RH, UK. E-mail: n.clarke@sheffield.ac.uk

${ }^{b}$ Department of Materials Science and Engineering, University of Pennsylvania, Philadelphia, Pennsylvania, 19104, USA

$\dagger$ Electronic supplementary information (ESI) available. See DOI: $10.1039 / \mathrm{c} 4 \mathrm{sm} 01980 \mathrm{f}$ nanoparticle dispersion ${ }^{3,4}$ can have an important effect on the polymer chain dimensions and this depends on the nanoparticle-polymer interaction, nanoparticle-polymer size ratio, ${ }^{8}$ size of nanoparticles and nanoparticle volume fraction. In the recent study of $\mathrm{PS} /$ silica, ${ }^{5}$ where the nanoparticles $(R=6.5 \mathrm{~nm})$ were well dispersed, no changes in polymer dimensions were observed. However we need to note that in the experimental area of polymer nanocomposites containing spherical nanoparticles, the role of monomer-nanoparticle interactions on polymer conformations is not yet clear. ${ }^{9}$

Nevertheless, by using the self-consistent polymer reference interaction site model (SC/PRISM), ${ }^{9}$ it was observed that spherical nanoparticles, smaller than the polymer chains and attracted to them, perturbed the polymer chain dimensions. The nanoparticles cause an increase in the radius of gyration with an increase in the nanoparticle volume fractions in accordance to the (PS) nanoparticle system, ${ }^{2,8}$ although there are significant differences between the theoretical model system and the experimental one. Some of the expansion is due to the effects of the excluded volume created by the nanoparticles, the nanoparticles act as good solvents to swell the polymers. From a simulation point of view, there is also controversy as to whether the addition of attractive nanoparticles to a polymer melt causes polymer chains either to expand, ${ }^{\mathbf{1 0 - 1 4}}$ remain unaltered $^{\mathbf{1 5 - 2 0}}$ or reduce their dimensions $^{21-23}$ compared to their size in the bulk. While most of these simulation studies were performed for low volume fraction of filler ${ }^{12-14,16,17,23}$ and for nanoparticle size similar to the polymer size, ${ }^{12,14,16}$ it is well recognized ${ }^{3,5-8}$ that in the case of polymernanoparticles mixtures, the polymer dimensions can be influenced by the characteristics of the nanoparticles (e.g. size, type of nanoparticle surface, dispersion, volume fraction of nanoparticles). To the best of our knowledge there are no studies that have addressed polymer dimensions in nanocomposites above 
the percolation threshold $\left(\phi_{\mathrm{c}}=31 \%{ }^{24}\right)$, except the work of Vacatello $^{22}$ that was implemented at constant density and for spatially frozen nanoparticles of size $R=4-8 \mathrm{~nm}$.

The rest of this paper is organized as follows. In Section II, we present the general features of the simulation methodology and the simulation details that were used to investigate the polymers in melts and nanocomposites. Subsequently, in Section III, we investigate first the polymer and nanoparticle structure and secondly we calculate the radius of gyration for both unentangled and entangled polymers in nanocomposites as a function of nanoparticle loading, monomer-nanoparticle interaction and nanoparticle size. Finally in Section IV, conclusions are presented.

\section{Simulations methodology}

To address this fundamental question we use the molecular dynamics method ${ }^{25-28}$ of a Kremer-Grest model. ${ }^{29}$ The classical Newton-Langevin equations that govern the motion of the particles is: $:^{25,29}$

$$
m_{i} \frac{\mathrm{d} \mathbf{u}_{i}}{\mathrm{~d} t}=-\nabla V_{i}-\Gamma \frac{\mathrm{d} \mathbf{r}_{i}}{\mathrm{~d} t}+\mathbf{W}_{i}(t)
$$

where is $V_{i}$ is the potential acting experienced by particle $i$ and $m_{i}$ is its mass. $\Gamma$ is the friction coefficient and $\mathbf{W}_{i}$ describes the random force which essentially is a Gaussian white noise with zero mean acting on each particle. The total force on particle $i$, $\mathbf{f}_{i}$, is the gradient of the potential $V_{i}$ given by a sum of three terms:

$$
V_{i}=\sum_{j \neq i}\left(V_{i j}^{\mathrm{LJ}}+V_{i j}^{\mathrm{S}}+V_{i j}^{\mathrm{B}}\right)
$$

The Lennard-Jones (LJ) potential $V_{i j}^{\mathrm{LJ}}$, acting along the line between the centres of mass of two particles force ${ }^{30}$ is given by:

$$
V_{i j}^{\mathrm{LJ}}=4 \varepsilon_{i j}\left(\frac{\sigma^{12}}{r_{i j}^{12}}-\frac{\sigma^{6}}{r_{i j}{ }^{6}}\right)
$$

where $\varepsilon_{i j}$ is the interaction energy between particle $i$ and particle $j$ (for monomers: $\varepsilon_{\mathrm{m}}=\sigma_{\mathrm{m}}=m_{\mathrm{m}}=1$, for nanoparticles: $\varepsilon_{\mathrm{p}}=1, \sigma_{\mathrm{p}}$ $\left.=2 R, R / \sigma_{\mathrm{m}}=1-4, m_{\mathrm{p}}=0.85 \frac{4}{3} \pi R^{3}\right)$, and $r_{i j}$ represents the distance between particles $i$ and $j$. For monomer (m)-nanoparticle $(\mathrm{p})$ interactions the combination rules: $\varepsilon_{\mathrm{mp}}=\left(\varepsilon_{\mathrm{m}} \varepsilon_{\mathrm{p}}\right)^{1 / 2}$, $\sigma_{\mathrm{mp}}=\left(\sigma_{\mathrm{m}}+\sigma_{\mathrm{p}}\right) / 2$ were used. ${ }^{30}$ The monomers (or nanoparticles) are modeled with the repulsive only part of eqn (3), shifted and truncated with a cut off radius at $r_{\mathrm{c}}=2^{1 / 6} \sigma_{\mathrm{m}}\left(r_{\mathrm{c}}=2^{1 / 6} \sigma_{\mathrm{p}}\right.$ for nanoparticles). For attractive monomer-nanoparticle interaction (attractive nanoparticles) the cut off radius is $r_{\mathrm{c}}=2.4 \sigma_{\mathrm{mp}}$, and for repulsive monomer-nanoparticle interaction (repulsive nanoparticles) $r_{\mathrm{c}}=2^{1 / 6} \sigma_{\mathrm{mp}}$. Also, the monomers were connected using FENE potential: ${ }^{29}$

$$
V_{i j}^{\mathrm{S}}=-\frac{1}{2} k R_{0}{ }^{2} \ln \left(1-\frac{r^{2}}{{R_{0}}^{2}}\right)
$$

where the sum is over all particles $j$ to which particle $i$ is connected. The equilibrium bond length was set to $R_{0}=1.5$, and $k=30 .^{29}$

The stiffness of the polymer chains is controlled by a cosine harmonic bending potential, ${ }^{31}$ which acts on three consecutive beads along the chain. By increasing the intramolecular stiffness of the polymer chain the entanglement length is decreased to a value of $N_{\mathrm{e}} \approx 58$.

$$
V_{i j k}^{\mathrm{B}}=\frac{1}{2} k_{\theta}\left(\cos \theta_{i j k}-\cos \theta_{0}\right)^{2}
$$

where $\theta_{i j k}$ is the bending angle between three consecutive bonds. The equilibrium value $\theta_{0}=109.5^{\circ}$, and the bending constant $k_{\theta}=25 .^{31}$

The simulations of the polymer melt were performed at a monomer density $\rho^{*}=N_{\mathrm{t}} / V=0.85$ in a simulation cell of total $N_{\mathrm{t}}=60000$ monomers in the simulation box (48 000 monomers were used for $N=80,160$ polymers), using the NVT ensemble. For equilibration of polymer melts the fast push off method was applied. $^{32}$ The pressure calculated for the $N=200$ polymer melt was $P^{*}=P \sigma_{\mathrm{m}}{ }^{3} / \varepsilon_{\mathrm{m}}=4.864$. That pressure was used to perform all the nanocomposite systems simulations in the NPT ensemble. The length of the simulation cell was always larger than the end-to-end distance of the polymer chains. To set the temperature at $T^{*}=\frac{k_{\mathrm{B}} T}{\varepsilon}=1$ and pressure at $P^{*}=4.864$ the Langevin thermostat with a friction constant $\Gamma=0.5 \tau^{-1}$ and the Berendsen barostat were used with time constant $\tau_{\mathrm{p}}=2 \tau$, respectively. The equations of motion were integrated using the Leap frog algorithm ${ }^{33}$ with a time step equal to $0.005 \tau$ for polymer melts (a time step of $0.004 \tau$ was used for nanocomposite simulations), where $\tau=\left(m \sigma_{\mathrm{m}}{ }^{2} /\left(k_{\mathrm{B}} T\right)\right)^{1 / 2}$ is the LJ time unit.

For nanocomposites, we consider systems of spherical nanoparticles in a dense polymer melt. The starting structures were created by an ensemble of polymers with $N=200$ and nanoparticles inserted at random positions within a large simulation box. Subsequently, the NPT ensemble was used at melt pressure of $P^{*}=4.864$ to gradually squeeze the simulation box to a dense polymer melt. The equilibration time is long enough for the polymer to move more than twice the radius of gyration of the bulk polymer, $R_{\mathrm{g}}$, which is the standard equilibration criterion..$^{16,34}$ Specifically, for polymer matrices $N=200$, the equilibrium time was $6 \times 10^{5} \tau \approx 3 \tau_{\mathrm{R}}$ (where $\tau_{\mathrm{R}}=2 \times 10^{5} \tau$ is the Rouse time for $N=350$ semiflexible polymers ${ }^{35}$ ) which is sufficient time to evolve the entanglement density. ${ }^{36}$ All the types of nanoparticles have reached their diffusive regime (where the mean square displacement of nanoparticles scales linearly with time). The duration of the simulation production runs were between $0.5-3.5 \times 10^{5} \tau$ depending on the length of molecules and nanoparticles. In the nanocomposite systems studied, a total number of $N_{\mathrm{t}}=23600$ monomers were used in a cubic cell for systems with nanoparticles radius of $R / \sigma_{\mathrm{m}}=1$ and $R / \sigma_{\mathrm{m}}=2$, and $N_{\mathrm{t}}=9440$ monomers for nanoparticle radius of $R / \sigma_{\mathrm{m}}=4$. The nanoparticle volume fraction, ${ }^{37}$ $\phi_{\mathrm{p}}=\frac{\sigma_{\mathrm{P}}{ }^{3} N_{\mathrm{P}}}{\sigma_{\mathrm{P}}{ }^{3} N_{\mathrm{P}}+\left(\sigma_{\mathrm{m}}{ }^{3} N_{\mathrm{t}} / 0.85\right)}$ is set by varying the number of 
Table 1 Nanoparticle volume fraction (\%), number of nanoparticles $N_{p}$, average radius of nanoparticles $R$ for nanocomposite systems

\begin{tabular}{lcccc}
\hline & $\frac{N_{\mathrm{p}}}{}$ & & $\frac{N_{\mathrm{p}}}{N_{\mathrm{p}}}$ \\
\hline volume fraction $\%$ & $R=1$ & $R=2$ & $R=4$ \\
\hline 10.3 & 400 & - & - \\
18.7 & 800 & 100 & 5 \\
25.7 & 1200 & - & - \\
31.6 & 1600 & 200 & 10 \\
40.9 & 2400 & 300 & 15
\end{tabular}

particles, $N_{\mathrm{p}}$. Details of the nanocomposite systems studied (nanoparticle volume fraction: $\phi(\%)$, number of nanoparticles: $N_{\mathrm{p}}$ of radius $R$ ) are summarized in Table 1.

\section{Results and discussion}

\section{A. Polymer and nanoparticle structure}

We first focus on the analysis of local polymer structure in nanocomposites. In Fig. 1 we show the monomer-nanoparticle radial distribution function $g_{\mathrm{mp}}(r)$ for different nanoparticle loading with attractive or repulsive small nanoparticles $(R=1)$ respectively. As can be seen from Fig. $1, g_{\mathrm{mp}}(r)$ exhibits a threelayer structure. The high monomer density of the layers establish a well defined interface between nanoparticles and polymer melt whose structure differs from that of the amorphous polymer melt. By dispersing attractive nanoparticle in the polymer matrix the polymer density around the nanoparticles increases as can be seen by the enhanced first peak of $g_{\mathrm{mp}}(r)$, comparing with $g_{\mathrm{mp}}(r)$ of repulsive nanoparticles (inset in Fig. 1). Also, the nanoparticle loading increases the monomer density of polymers in contact with the nanoparticle surface. Similar

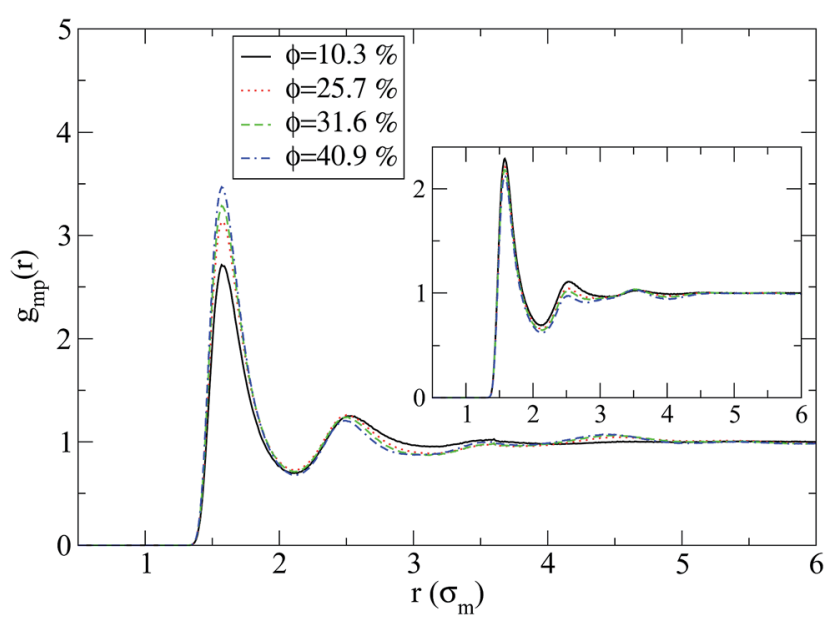

Fig. 1 Monomer-nanoparticle radial distribution functions in the nanocomposites at different volume fractions for attractive nanoparticles $(R=1)$ for $N=100$ : (i) $10.3 \%$ (black line), (ii) $25.7 \%$ (red line), (iii) $31.6 \%$ (green line), (iv) $40.9 \%$ (blue line). Inset: monomer-nanoparticle radial distribution functions in the nanocomposites for repulsive nanoparticles $(R=1)$ for $N=100$.

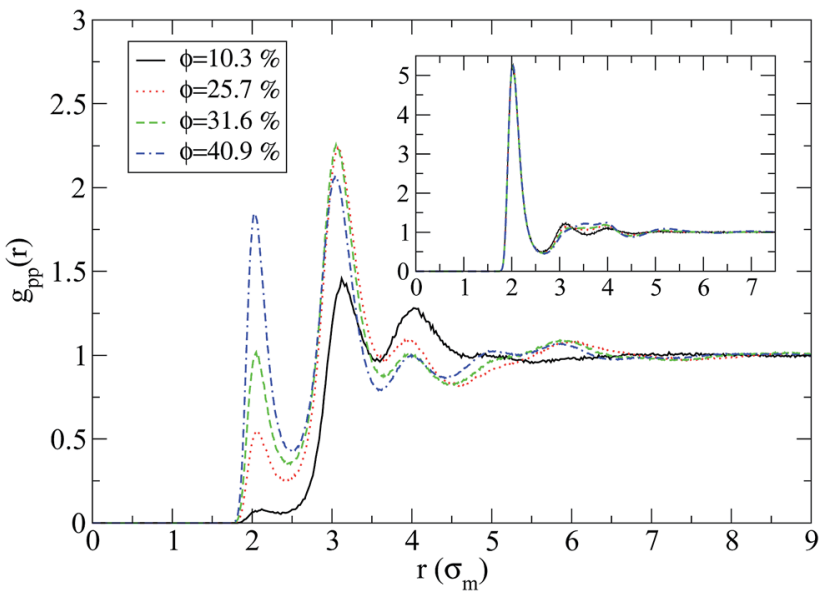

Fig. 2 Nanoparticle-nanoparticle radial distribution functions in the nanocomposites at different volume fractions for attractive nanoparticles $(R=1)$ for $N=100$ : (i) $10.3 \%$ (black line), (ii) $25.7 \%$ (red line), (iii) $31.6 \%$ (green line), (iv) $40.9 \%$ (blue line). Inset: nanoparticle-nanoparticle radial distribution functions in the nanocomposites with repulsive nanoparticles $(R=1)$ for $N=100$.

behaviour appears in nanocomposites containing larger nanoparticles $(R=2-4)$ (results not shown).

Regarding the nanoparticle structure an entire different behaviour for the two nanocomposite systems is observed as can be seen in Fig. 2. On one hand, when there is a repulsive monomer-nanoparticle interaction, there is a higher probability for the nanoparticles to be in contact with each other than in contact with monomers (inset of Fig. 2), while on the other hand attractive monomer-nanoparticle interaction leads the nanoparticles to be well dispersed in the polymer matrix. If the nanoparticle volume fraction is increased, there are more nanoparticle-nanoparticle contacts as can be seen from the first peak of $g_{\text {pp }}(r)$ in Fig. 2. By increasing the nanoparticle radius to $R=2$ or $R=4$, poor dispersion is observed for repulsive nanoparticles, especially in lowest nanoparticle loading $(\phi=$ $18.7 \%$ ) the nanoparticles aggregate to one big cluster as can be seen by the high intensity first peak of $g_{\mathrm{pp}}(r)$ in the inset of Fig. 3. Thus, in nanocomposites containing nanoparticles of radius to $R=2$ or $R=4$ the nanoparticles form aggregated clusters for repulsive monomer-nanoparticle interaction. Aggregation has been observed experimentally for polymer nanocomposites with weak interactions such as polystyrenesilica nanocomposite ${ }^{3,4}$ and possibly for the repulsive nanoparticle nanocomposite such as PEP-silica ${ }^{6}$ in which the TEM data were not reported. However, when the monomer-nanoparticle attraction is present there are no nanoparticle contacts for all the nanoparticles loading as can be seen from $g_{\mathrm{pp}}(r)$ in Fig. 3. The nanoparticles are well dispersed in the polymer melt (the same behaviour is observed for nanoparticles of radius $R=$ 4 - results not shown) in agreement with experimental observations for miscible systems such as PS chains-crosslinked PS nanoparticles. ${ }^{2}$

Attractive nanoparticles of radius $R=1$ have diffused in the polymer matrix $(N=200)$ several times of $R_{\mathrm{g}}$ distance at high nanoparticle loading $(\phi=40.9 \%)$ and its diffusivity is $D=4.4 \times$ 


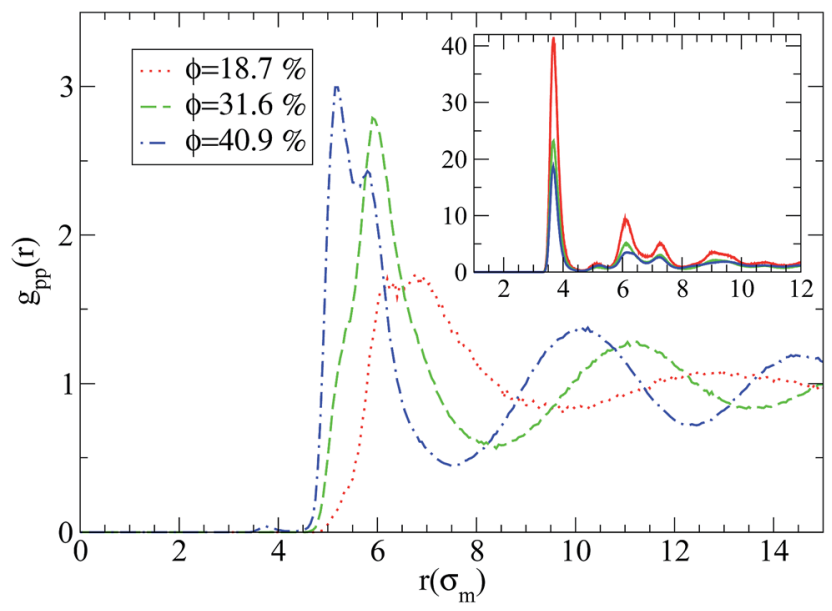

Fig. 3 Nanoparticle-nanoparticle radial distribution functions in the nanocomposite systems at different volume fractions for attractive nanoparticles $(R=2)$ for $N=100$ : (i) $18.7 \%$ (red line), (ii) $31.6 \%$ (green line), (iii) $40.9 \%$ (blue line). Inset: nanoparticle-nanoparticle radial distribution functions in the nanocomposites with repulsive nanoparticles $(R=2)$ for $N=100$.

$10^{-4}\left(\sigma_{\mathrm{m}}{ }^{2} / \tau\right)$. Thus, we can consider them as mobile. The diffusivity of large attractive nanoparticles $(R=2,4)$ is small though due to their high mass, for example at $\phi=40.9 \%$ (polymer matrics: $N=200$ and $N=160$ respectively), their diffusivity is $D$ $=2.7 \times 10^{-5}$ (for $R=2$ ) and $D=4.39 \times 10^{-6}\left(\sigma_{\mathrm{m}}{ }^{2} / \tau\right)$ (for $R=4$ ). Since the large nanoparticles of $R=2,4$ have not diffused a distance further than twice their diameter at high nanoparticle loading, in an entangled matrix, we consider them as immobile (see mean square displacement of nanoparticles $R=2$ in the ESI $\dagger$ section).

\section{B. Polymer dimensions}

We now focus on the polymer dimensions analysis on melts and nanocomposites. The radius of gyration of a molecule, which is defined as the average squared distance between monomers in given conformation and the molecule's center of mass is given by: ${ }^{38,39}$

$$
\left\langle R_{\mathrm{g}}^{2}(N)\right\rangle=\frac{1}{N}\left\langle\sum_{i=1}^{N}\left(\mathbf{r}_{i}-\mathbf{r}_{\mathrm{cm}}\right)^{2}\right\rangle
$$

where $\mathbf{r}_{\mathrm{cm}}=\frac{1}{N} \sum_{i=1} \mathbf{r}_{i}$ is the center of mass of the chain. The radii of gyration of the polymer melt simulated systems are given in Table 2 .

Let's first focus on polymer dimensions of nanocomposites with nanoparticles $(R=2)$ dispersed in polymer matrix $(N=$

Table $2 N$, number of monomers per polymer chain, $R_{\mathrm{go}}\left(\sigma_{\mathrm{m}}\right)$ average radius of polymer gyration for polymer melt systems studied in the present simulations

\begin{tabular}{lllllllll}
\hline$N$ & 10 & 20 & 40 & 50 & 80 & 100 & 160 & 200 \\
\hline$R_{g 0}$ & 1.577 & 2.367 & 3.479 & 3.915 & 5.019 & 5.626 & 7.211 & 8.05
\end{tabular}

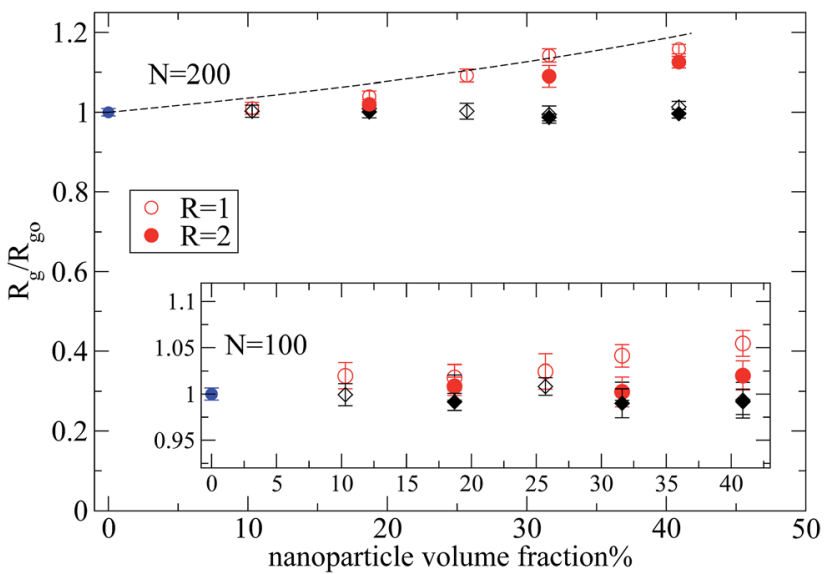

Fig. 4 Radius of gyration of polymers in melt with nanoparticles of radius $R=1,2$ normalized with its value in the bulk, for $N=200$ and $N$ $=100$ (inset): (i) polymer melt (blue filled circles), (ii) nanocomposite: attractive monomer-nanoparticle $(R=2)$ interactions (red filled circles), (iii) nanocomposite: repulsive monomer-nanoparticle $(R=2)$ interactions (black filled diamonds), (iv) nanocomposite: attractive monomer-nanoparticle $(R=1)$ interactions (red open circles), (v) nanocomposite: repulsive monomer-nanoparticle $(R=1)$ interactions (black open diamonds). The black dashed line shows $R_{\mathrm{g}} / R_{\mathrm{g} 0}=$ $(1-\phi)^{-1 / 3}$.

100, 200). The trend is different between the two types of nanocomposites as can be seen in Fig. 4. In nanocomposites containing repulsive nanoparticles (black symbols), the polymers dimensions are not altered by the nanoparticle loading. The polymers are phase separated from the repulsive nanoparticles (of $R=2$ ) in the nanocomposites, thus there is no change on radius of gyration values. On the other hand, in the nanocomposites containing attractive nanoparticles, the $R_{\mathrm{g}}$ increases, with increased nanoparticle loading, compared to its bulk value.

By reducing the nanoparticle radius of the nanocomposite to $R=1$, similar trends can be observed as can be seen in Fig. 4 . In this case, even though there are smaller clusters (inset of Fig. 2) than in the nanocomposites with large nanoparticles, there is no polymer swelling. When attractive monomer-nanoparticle interactions are present the overall polymer dimensions increase dramatically at high nanoparticle loading. In particular, the magnitude of expansion of polymer dimensions is larger for polymers with $N=200$ following qualitatively the experimental data. ${ }^{7,8}$ In Fig. 4 we depict the relation $R_{\mathrm{g}} / R_{\mathrm{g} 0}=(1$ $-\phi)^{-1 / 3}$ (ref. 9) which predicts the polymer expansion due to the excluded volume introduced by the nanoparticles, assuming no change in density on mixing. The end-to-end distance data follow the same trend. All the polymers have a Gaussian conformation up to percolation threshold for all the nanocomposites. In nanocomposites $(N=160-200)$ above the percolation threshold the ratio $\left\langle R_{\mathrm{ee}}\right\rangle^{2} /\left\langle R_{\mathrm{g}}\right\rangle^{2} \approx 6.1-6.22$, so, we consider that the polymers still keep their Gaussian conformation. We also report the mean square internal distances for nanocomposite systems containing small nanoparticles $(R=1)$ in the ESI $\uparrow$ section (data for larger nanoparticles have similar trends). 
Hence polymer expansion appears in nanocomposites with attractive interactions and increases with the nanoparticle loading, the very small nanoparticles such as of radius $R=1$ act as a good solvent and cause the polymer to expand. To further characterize the polymer structure, we calculated the principal moments (eigen values) of the gyration tensor, $\lambda^{2}{ }_{i(i=1-3)}$ (where $\left.R_{\mathrm{g}}{ }^{2}=\lambda_{1}{ }^{2}+\lambda_{2}{ }^{2}+\lambda_{3}{ }^{2}\right)$, for different nanoparticles volume fractions and depict these in Fig. 5. In our simulations for a polymer melt with chains of $N=200$, the principal moments of the gyration tensor approach, $\lambda_{1}{ }^{2}: \lambda_{2}{ }^{2}: \lambda_{3}{ }^{2}=12.1: 2.7: 1 .^{40}$ At high nanoparticle loading, $\phi \geq 18.7 \%$, the polymer chains are stretched and flattened by the nanoparticles, as can be seen from the $\lambda_{1}{ }^{2}, \lambda_{2}{ }^{2}$ values in comparison to the bulk values. Even oligomers $(N=20-50)$ are stretched and flattened at the highest nanoparticle loading (results not shown).

In the case of nanocomposites containing short polymers $(N=10,20,50)$ as matrix and repulsive nanoparticles, polymers remain unaltered compared with their melt values as can be seen in Fig. 6. Instead when attractive interactions are present the polymer dimensions increase compared with its melt value for small nanoparticles and loading $\phi \geq 18.7 \%$ even for the smallest polymers studied $(N=10)$ (where $R_{\mathrm{g}}>R$ ). This polymer expansion with nanoparticle loading becomes more abrupt for larger polymers $(N=50)$.

Then, we focus on the analysis of a nanocomposite system with larger nanoparticles of $R=4$, in which the $R_{\mathrm{g}}$ of polymers chains with $N=160\left(N / N_{\mathrm{e}} \approx 3\right)$ monomers is of the order of nanoparticle diameter $\left(\left(\frac{R_{\mathrm{g}}}{R} \approx 1.7\right)\right.$, which is made equivalent to the experimental repulsive nanocomposite by mixing PEP and hydrophobic modified silica. ${ }^{6}$ By such an increase of the nanoparticle radius at a constant nanoparticle volume fraction, we decrease the interfacial area. For the case of polymers $(N=80,160)$ and repulsive nanoparticles the $R_{\mathrm{g}}$

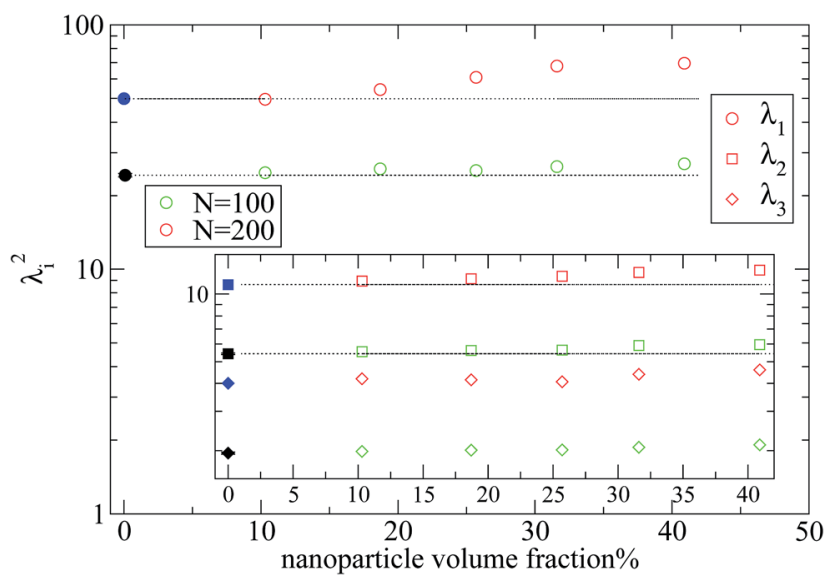

Fig. 5 Principal moments (eigenvalues) of the gyration tensor, $\lambda_{i}{ }^{2}$, in nanocomposites with attractive nanoparticles of radius $R=1$, for polymers $N=100-200$, at different volume fractions: (i) polymer melt: $N=200$ (blue filled symbols), (ii) polymer melt: $N=100$ (black filled symbols), (iii) nanocomposite: $N=200$ (red open symbols), (iv) nanocomposite: $N=100$ (green open symbols). The dashed lines are guide to the eye. The error bars are smaller than the symbol size.

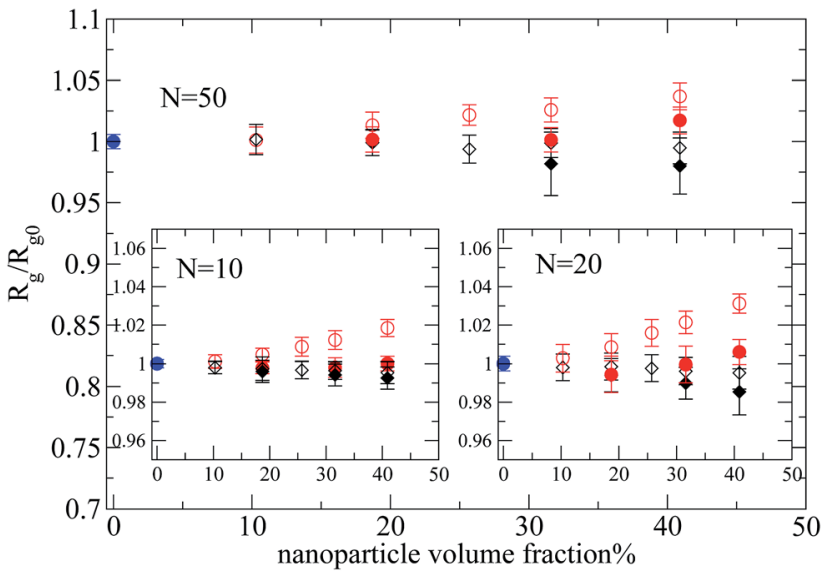

Fig. 6 Radius of gyration of short polymers in melt and nanocomposite $(R=1,2)$ normalized with its value in the bulk, for different number of monomers per chain $(N=10,20,50)$ : (i) polymer melt (blue filled circles), (ii) nanocomposite: attractive monomer-nanoparticle $(R$ $=2$ ) interactions (red filled diamonds), (iii) nanocomposite: repulsive monomer-nanoparticle $(R=2)$ interactions (black filled diamonds), (iv) nanocomposite: attractive monomer-nanoparticle $(R=1)$ interactions (red open circles), (v) nanocomposite: repulsive monomer-nanoparticle $(R=1)$ interactions (black open circles).

remains unperturbed for all the nanoparticle loadings. Even for high nanoparticle loading we can not see any polymer contraction as was observed for entangled PEP polymers filled with silica nanoparticles ${ }^{6}$ (where there is a polymer contraction of $12 \%$ above percolation $(\phi=50 \%))$. This may arise either from the fact that in the large nanoparticles $(R=4)$ nanocomposites studied $\frac{R_{\mathrm{g}}}{R} \approx 1.225-1.85$, whereas in Nusser's work $\frac{R_{\mathrm{g}}}{R} \approx 1$ (ref. 6) or that the volume fraction studied is not high enough since above the percolation

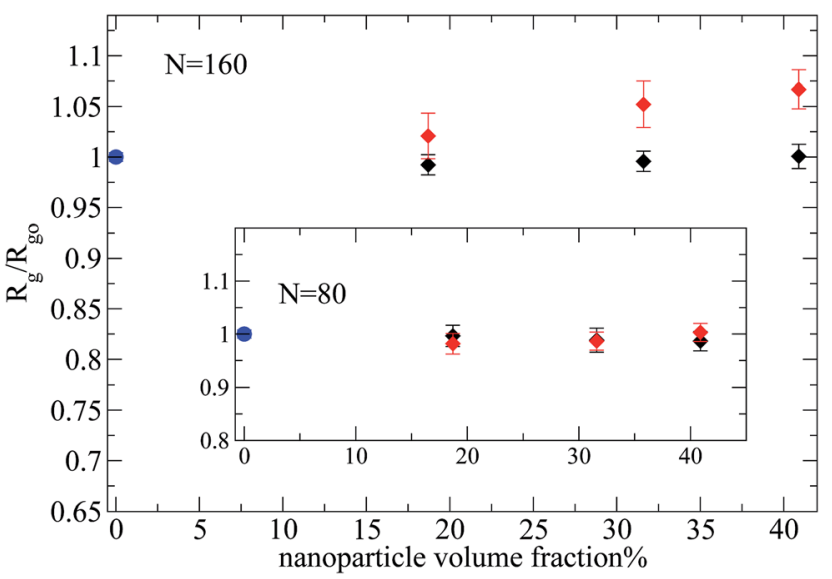

Fig. 7 Radius of gyration of polymers in melt and nanocomposites ( $R$ $=4$ ) normalized with its value in the bulk, for different number of monomers per chain ( $N=160$ and $N=80$ (inset)): (i) polymer melt (blue filled circles), (ii) nanocomposite: attractive monomer-nanoparticle interactions (red diamonds), (ii) nanocomposite: repulsive monomer-nanoparticle interactions (black diamonds). 
threshold $\left(\phi_{\mathrm{c}} \approx 31 \%\right)$ with increasing $\phi$ the geometrical confinement strongly gains importance. ${ }^{6}$ However, the monomer nanoparticle attraction does alter distinctively the polymer dimensions especially for high nanoparticle volume fraction as can be seen in Fig. 7. At nanoparticle loading $\phi=$ $18.7 \%$ the polymer chains remain unperturbed in agreement with the weakly interacting PS/silica nanocomposite ${ }^{5}$ which was investigated up to $\phi=32 \%$ nanoparticle loading. Additionally, we have observed that the average radius of gyration of the short polymer chains $(N=10,20,40)$, in nanocomposites containing large nanoparticles of $R=4$, does not change compared to its melt value for both repulsive and attractive nanoparticles at all volume fractions studied (results not shown). Thus we can conclude that short polymers in nanocomposites with $R_{\mathrm{g}} \leq R$, remain unperturbed as this result has also been observed experimentally in the PEP/ silica nanocomposites. ${ }^{6,41}$

\section{Conclusions}

To summarize, we investigated the conformations of polymers, for the first time in nanocomposites containing small spherical nanoparticles at high volume fraction, as depicted on the polymer radius of gyration in a broad size range 1.225 $\leq R_{\mathrm{g}} / R \leq 7.85$ (for $N=80-200$ ) for a range of nanoparticle loadings using molecular dynamics simulations. We find that in nanocomposites with repulsive monomer-nanoparticle interaction the polymers do not contract when taking into account the error margin. On the other hand, in nanocomposites with attractive monomer-nanoparticle interactions the polymers increase their size with the nanoparticle loading in qualitative agreement with the experimental data. ${ }^{2,7}$ We showed that in such nanocomposites with well dispersed small nanoparticles, the condition $R_{g}>R$ is essential for polymer swelling in agreement with the observations of Nakatani ${ }^{7}$ and Tuteja-Mackay. ${ }^{2}$ In particular, this behaviour becomes more dramatic for the systems with the smallest nanoparticles $(R=1)$ dispersed in the polymer matrix where even short polymers can increase their dimensions slightly with nanoparticle loading when $R_{\mathrm{g}}>R$, due to the high interfacial area following the observations of Nakatani ${ }^{7}$ and Tuteja-Mackay. ${ }^{2}$ Also, the fact that small nanoparticles of $R=1$ are very mobile can also contribute on the chain expansion as reported in. ${ }^{42}$ In addition, the short polymer chains remain unperturbed from the nanoparticles when $R_{\mathrm{g}} \leq R$, in agreement with the experimental data. ${ }^{6}$

In experiments only the nanocomposites systems by Naka$\operatorname{tani}^{7}$ and Tuteja ${ }^{2}$ contain very small nanoparticles of $R=1 \mathrm{~nm}$ and $R=2 \mathrm{~nm}$, whereas in the work of $\mathrm{Kumar}^{3,5}$ in which no polymer expansion was observed, the nanoparticle size was $R=$ $6.5 \mathrm{~nm}$ although the polymer radius of gyration was larger than $R$. Thus, we can conclude that the polymer-nanoparticle interaction, nanoparticle size and nanoparticle loading can play a major role in the polymer radius of gyration for nanocomposites in the size range of $\frac{R_{g}}{R} \geq 1$ and for very small nanoparticles which are well dispersed in the polymer matrix.

\section{Acknowledgements}

This research was funded by the EPSRC/NSF Materials Network program EP/5065373/1 (EPSRC:NC, AK) and DMR-1210379 (NSF:KIW, RJC).

\section{References}

1 S. K. Kumar and R. Krishnamoorti, Annu. Rev. Chem. Biomol. Eng., 2010, 1, 37.

2 A. Tuteja, P. Duxbury and M. Mackay, Phys. Rev. Lett., 2008, 100, 077801.

3 S. Sen, Y. Xie, S. K. Kumar, H. Yang, A. Bansal, D. L. Ho, L. Hall, J. B. Hooper and K. S. Schweizer, Phys. Rev. Lett., 2007, 98, 128302.

4 N. Jouault, F. Dalmas, S. Said, E. Di Cola, R. Schweins, J. Jestin and F. Boue, Macromolecules, 2010, 43, 9881.

5 M. K. Crawford, R. J. Smalley, G. Cohen, B. Hogan, B. Wood, S. K. Kumar, Y. B. Melnichenko, L. He, W. Guise and B. Hammouda, Phys. Rev. Lett., 2013, 110, 196001.

6 K. Nusser, S. Neueder, G. J. Schneider, M. Meyer, W. Pyckhout-Hintzen, L. Willner, A. Radulescu and D. Richter, Macromolecules, 2010, 43, 9837.

7 A. Nakatani, W. Chen, R. Schmidt, G. Gordon and C. Han, Polymer, 2001, 42, 3713.

8 M. E. Mackay, A. Tuteja, P. M. Duxbury, C. J. Hawker, B. Van Horn, Z. Guan, G. H. Chen and R. S. Krishnan, Science, 2006, 311, 1740.

9 A. L. Frischknecht, E. S. McGarrity and M. E. Mackay, J. Chem. Phys., 2010, 132, 204901.

10 F. W. Starr, T. B. Schroder and S. C. Glotzer, Phys. Rev. E: Stat., Nonlinear, Soft Matter Phys., 2001, 64, 021802.

11 J. Huang, Z. Mao and C. Qian, Polymer, 2006, 47, 2928.

12 M. Goswami and B. G. Sumpter, Phys. Rev. E: Stat., Nonlinear, Soft Matter Phys., 2010, 81, 041801.

13 G. G. Voyiatzis, E. Voyiatzis and D. N. Theodorou, Eur. Polym. J., 2011, 47, 699.

14 T. V. M. Ndoro, E. Voyiatzis, A. Ghanbari, D. N. Theodorou, M. C. Bohm and F. Müller-Plathe, Macromolecules, 2011, 44, 2316.

15 G. D. Smith, D. Bedrov, L. Li and O. Byutner, J. Chem. Phys., 2002, 117, 9478.

16 T. Desai, P. Keblinski and S. K. Kumar, J. Chem. Phys., 2005, 122, 134910.

17 P. J. Dionne, R. Osizik and C. R. Picu, Macromolecules, 2005, 38, 9351.

18 A. Karatrantos, R. J. Composto, K. I. Winey and N. Clarke, Macromolecules, 2011, 44, 9830.

19 A. Karatrantos, R. J. Composto, K. I. Winey, M. Kröger and N. Clarke, Macromolecules, 2012, 45, 7274.

20 A. Karatrantos, N. Clarke, R. J. Composto and K. I. Winey, Soft Matter, 2013, 9, 3877.

21 M. Vacatello, Macromolecules, 2001, 34, 1946.

22 M. Vacatello, Macromolecules, 2002, 35, 8191.

23 M. Vacatello, Macromol. Theory Simul., 2003, 12, 86.

24 M. J. Powell, Phys. Rev. B: Condens. Matter Mater. Phys., 1979, 20, 4194. 
25 H. Bekker, H. J. C. Berendsen, E. J. Dijkstra, S. Achterop, R. van Drunen, D. van der Spoel, A. Sijbers, H. Keegstra, B. Reitsma and M. K. R. Renardus, Physics Computing 92, 1993, 252.

26 H. J. C. Berendsen, D. van der Spoel and R. van Drunen, Comput. Phys. Commun., 1995, 91, 43.

27 E. Lindahl, B. Hess and D. van der Spoel, J. Mol. Model., 2001, 7, 306.

28 D. van der Spoel, E. Lindahl, B. Hess, G. Groenhof, A. Mark and H. Berendsen, J. Comput. Chem., 2005, 26, 1701.

29 K. Kremer and G. S. Grest, J. Chem. Phys., 1990, 92, 5057.

30 M. P. Allen and D. J. Tildesley, Computer Simulation of Liquids, Clarendon Press, Oxford, 1987.

31 M. Bulacu and E. van der Giessen, J. Chem. Phys., 2005, 123, 114901.

32 R. Auhl, R. Everaers, G. S. Grest, K. Kremer and S. J. Plimpton, J. Chem. Phys., 2003, 119, 12718.

33 W. F. van Gunsteren and H. C. J. Berendsen, Mol. Simul., 1988, 1, 173.
34 J. Liu, Y. Wu, J. Shen, Y. Gao, L. Zhang and D. Cao, Phys. Chem. Chem. Phys., 2011, 13, 13058.

35 M. Pütz, K. Kremer and G. S. Grest, Europhys. Lett., 2000, 49, 735.

36 R. S. Hoy and M. O. Robbins, Phys. Review E, 2005, 72, 061802.

37 J. T. Kalathi, G. S. Grest and S. K. Kumar, Phys. Rev. Lett., 2012, 109, 198301.

38 P. Flory, Statistical Mechanics of Chain Molecules, Hanser Publishers, Munich, 1989.

39 M. Rubinstein and R. H. Colby, Polymer Physics, Oxford University Press, New York, 2003.

40 R. C. Picu and M. S. Ozmusul, J. Chem. Phys., 2003, 118, 11239.

41 K. Nusser, G. I. Schneider, W. Pyckhout-Hintzen and D. Richter, Macromolecules, 2011, 44, 7820.

42 F. M. Erguney, H. Lin and W. L. Mattice, Polymer, 2006, 47, 3689 . 